\title{
Stimulus presentation rate and verbal response efficiency
}

MUSETTA C. GORDON AND ALAN M. GORDON WOODWARD STATE HOSPITAL-SCHOOL

Sixty Ss, 20 in each of three IQ levels (Normal, Hi IQ Retarded and Lo IQ Retarded) responded with a single word each time they heard a tap, which was presented for half of the Ss once every $2 \mathrm{sec}$ and for the other half once every 4 sec. The Hi IQ Retarded Ss' performance was like the Lo IQ Retarded group at the faster presentation rate but like the Normal Ss at the slower rate. Efficiency of performance for all groups was greater at the slower rate.

That perception time is a critical variable in the study of short-term memory is amply shown in the review of the literature by Aaronson (1967). The present study seeks to demonstrate the necessity for considering perception time when measuring the efficiency of other cognitive functions.

The concept of an analyzer used in the Pavlovian reflex theory of sensation implies the dual processes of selection of essential components of stimuli and the inhibition of the nonessential, subsidiary components. Luria (1966) says, ". . . sensation incorporates the process of analysis and synthesis of signals while they are still in the first stages of arrival. . the units of any sensory process are not only acts of reception of individual signals measurable in terms of thresholds of sensation, but also acts of complex analysis and integration of signals, measurable in units of comparison and discrimination." He further states that both the perception of speech and the pronunciation of words are mediated by the same kinesthetic and auditory analyzers.

Fraisse (1963) suggests that patients with damaged brains or neurological disorders have difficulty in integrating successive bits of information because the perception of each element seems to develop independently and the process of perception takes longer for them than it does for persons with intact central nervous systems (CNS).

We infer that it is not only the subnormal structure of the organism, but also its faulty timing, which impairs performance. If we reset, so to speak, the rate of input, can we get greater efficiency of performance from persons with defective CNS mechanisms?

The temporal interval between two stimuli of a sequence is the critical variable. Rapidly presented stimuli might not elicit one for one responses from a person with a subnormal CNS if the perception of the stimulus and the integration of an appropriate response takes longer than in a normal person. Differential performances as a function of time between stimuli might also be obtained from within a subnormal population-those with less abnormality performing significantly better at a faster rate of stimulus presentation than those with greater abnormality, but not necessarily better at a slower rate.

Since there is the theoretical suggestion that both the perception of speech and the pronunciation of words (which we interpret to mean the production of words) are mediated by the same analyzers (Luria, 1966), tests of our hypotheses were made using verbal production of words as the dependent variable.

\section{Subjects}

Ss were 40 patients at the Woodward State Hospital-School for the retarded, who were divided into Low and High IQ groups on the basis of their FS WAIS. The range for the Low IQ group was 44-59 with a mean of 51.6, and the range for the High $\mathrm{IQ}$ group was 61-78 with a mean of 67.2. The two groups had mean CAs of 22.7 and 24.3 , respectively, a difference that was not significant. Twenty Ss from the secretarial and nursing staff of the VA Hospital in Des Moines comprised the intellectually normal group. These persons had no history of cerebral trauma or known periods of unconsciousness. Their mean $\mathrm{CA}$ was 25.7 and this did not differ significantly from the mean CAs of either group of retarded Ss. Procedure

To be certain that all Ss had responses available for the test tasks, each was asked to name as many words as he could in $1 \mathrm{~min}$. These responses were tape recorded and later transcribed and totaled.

The tests were administered directly after the $1 \mathrm{~min}$ free rate of responding task. Each group of 20 Ss was divided into two subgroups. Half of each IQ group was asked to name a word every time they heard a tap at the rate of 15 taps per min. The other half were asked to name a word each time they heard a tap at the rate of 30 taps per min. The words produced by each $\mathrm{S}$ for a full minute were recorded. The criterion scores were the total number of words produced by a $S$ at the rate condition which had been randomly assigned to him from within his $I / 2$ group. All Ss produced more words in the free rate of responding task than they did in the controlled rates of responding tasks. No S's supply of available responses was depleted during the controlled rate of responding.

Results

A Task Rate by IQ Levels ANOV was made of the scores. A significant interaction between Task and 


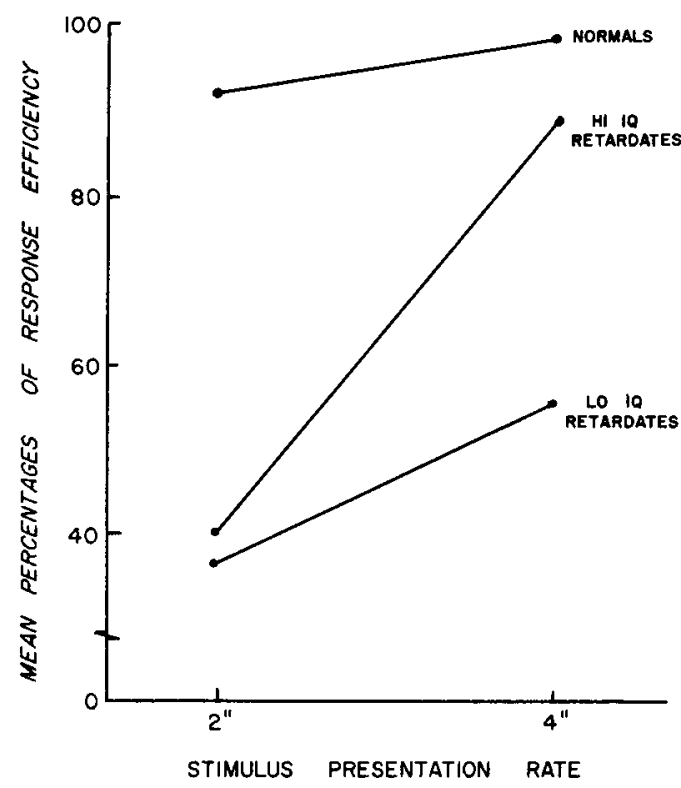

Fig. 1. Mean percentages of response efficiency for groups at two different stimulus presentation rates.

IQ Levels was revealed $(F=16.19, p<.001)$. Fig. 1 shows a graph of the mean percentages of possible responses made by each subgroup.

The High IQ Retarded group performed as poorly as the Low IQ Retarded group at the fast rate of production $(t=.30, p>.70)$, not significantly better as was predicted; yet they performed as well as the normal group at the slow rate of responding $(t=1.51$, $\mathrm{p}>.10)$.

It appeared that the High IQ retardates showed the most notable difference in performance at the two rates of stimulus presentation. Since word fluency is usually considered to be related to intelligence, it seemed advisable to check the two subgroups for equality of IQ. The random assignment of the small sample to the two treatment subgroups might have resulted in an error of distribution of levels of intellectual functioning within this particular level. Mean IQs for the $2 \mathrm{sec}$ and the $4 \mathrm{sec}$ rate subgroups were 66.4 and 68.0 , with standard deviations of 4.22 and 6.7 , respectively. $\mathrm{A} t$ test of the mean difference in two samples taken from the same population resulted in a $\mathrm{t}=1.91, \mathrm{p}>.05$.

While all three groups of Ss performed better at the slower rate of responding, it was the two groups of retarded Ss who exhibited significantly greater increases in ability to respond efficiently at the slower rate. For the High IQ Retarded group the $t$ was $4.41, p<.005$, and for the Low IQ Retarded group the $\mathrm{t}$ was $5.04, \mathrm{p}<.005$.

Discussion

The retarded Ss increased their efficiency of verbal production when stimuli were presented at a $4 \mathrm{sec}$ (slow) rate rather than at a $2 \mathrm{sec}$ (faster) rate. In particular, the High IQ Retarded group performed with an efficiency no different from the normal Ss at the slow rate of stimulus presentation. Their measured IQs suggest subnormal CNSs, but one wonders if their poorer performance at the faster rate of stimulus presentation could be due to faulty timing and/or faulty structure of neural mechanisms.

In general, the results support the hypothesis that subnormal nervous systems are unable to perceive, analyze, and integrate a stimulus-response sequence as rapidly or as efficiently as an intact nervous system is able to do. This contention has found support in many areas of investigation which involve other than verbal mediators, e.g., reaction time studies. It is well known that retardates have slower reaction times than do normals. The idea of a delayed perceptual development and integration time could also be enlisted to explain the lower than average critical flicker fusion rates found in retardates. If it takes longer for a stimulus unit to be focused by the inhibitors of unessential subsidiary components of the stimulus, the blurred unit or signal will still be in the process of being integrated and analyzed when another unit comes in over the same system, and there will be no discrimination between the two units.

An implication of the findings is that optimal stimulus presentation rates should be identified for training the retarded and the brain-damaged.

\section{References}

Aaronson, Doris. Temporal factors in perception and short-term memory. Psychol. Bull., 1967, 67, 130-144.

Fraisse, P. The psychology of time. New York: Harper \& Row, 1963.

Luria, A. R. Higher cortical functions in man. New York: Basic Books, Inc., 1966.

\section{Note}

1. Thanks are due Dr. S. J. Williamson, Jr., Chief of Psychology Service, Veterans Administration Hospital, Des Moines, Iowa, for his assistance in obtaining normal $S$ s for this study. 